\title{
La diffusion du sport en Guadeloupe : enjeux culturels autour de la départementalisation
}

\section{Jacques Dumont}

\section{(2) OpenEdition}

1 Journals

\section{Édition électronique}

URL : http://journals.openedition.org/corpsetculture/848

DOI : 10.4000/corpsetculture.848

ISSN : 1777-5337

\section{Éditeur}

Association Corps et Culture

\section{Édition imprimée}

Date de publication : 1 janvier 2004

ISSN : 1268-5631

\section{Référence électronique}

Jacques Dumont, « La diffusion du sport en Guadeloupe : enjeux culturels autour de la

départementalisation », Corps et culture [En ligne], Numéro 6/7| 2004, mis en ligne le 12 octobre 2007, consulté le 08 septembre 2020. URL : http://journals.openedition.org/corpsetculture/848 ; DOI :

https://doi.org/10.4000/corpsetculture.848

Ce document a été généré automatiquement le 8 septembre 2020.

(c) tous droits réservés 


\title{
La diffusion du sport en Guadeloupe : enjeux culturels autour de la départementalisation
}

\author{
Jacques Dumont
}

1 Etudier la diffusion du sport dans une petite colonie insulaire devenant département pose à l'évidence le problème des références culturelles, et indissolublement celui de leur médiation. La situation coloniale évoque l'acculturation, et suggère une mécanique d'imposition. Pourtant ce point de vue, en postulant la passivité des principaux protagonistes risque de négliger la dynamique culturelle, en particulier celle des réappropriations. Il semble préférable d'examiner des processus de transferts culturels (Turgeon L. et al., 1995), où des biens sont échangés dans le but de s'affirmer. Certes ces échanges, profondément déséquilibrés en situation de dépendance coloniale, peuvent relever d'un marché de dupes, mais ils sont à replacer aux Antilles dans une histoire complexe, où s'entremêlent processus d'assimilation, revendication égalitaire et affirmations identitaires.

2 L'abolition de l'esclavage en 1848, en octroyant la citoyenneté française à tous les habitants des "vieilles colonies ", place le phénomène d'assimilation au centre d'une quête. On sait depuis Tocqueville que l'expérience démocratique n'est pas seulement un mode politique, mais repose sur le fondement anthropologique de l'idée d'égalité. Or, celle-ci, proclamée devant la loi et inscrite sur le fronton des mairies, tarde à être mise en œuvre. D'une part, politiquement, les institutions ne sont pas équivalentes, ce qui va entrainer la forte demande de départementalisation, perçue comme une garantie, dont la loi du 19 mars 1946 constitue en principe l'aboutissement. D'autre part, socialement, les signes d'une intégration sont sans cesse différés. La conscription, par exemple, revendiquée par les députés antillais au titre de la participation aux devoirs de tout citoyen, et pourtant décrétée le 15 septembre 1889, ne sera appliquée - fort opportunément - qu'en 1913. L'école de la troisième République, vécue comme vecteur d'émancipation, n'accueille qu'avec peine la moitié de la population scolarisable en Guadeloupe $^{1}$ et ce jusqu'après la seconde guerre mondiale. 
3 L'assimilation, c'est-à-dire la fusion d'un ensemble d'individus dans un groupe plus large, est donc d'abord refusée ou différée, et de ce fait érigée en sésame ouvrant les portes d'une complète intégration. Or, pour accéder à cette " promotion ", le candidat doit être reconnu et pensé apte, c'est-à-dire considéré comme semblable. Le jeu entre l'utilisation ostensible de marques d'appartenance et la quête d'une reconnaissance est particulièrement sensible dans le phénomène sportif et invite à examiner les conditions et les effets d'une greffe, mais aussi la dynamique d'une redéfinition. De façon schématique deux temps peuvent être distingués. L'un de copie d'un modèle extérieur, l'autre d'affirmation d'une différence. L'ambition de cet article est pourtant de montrer que ces deux positions ne sont pas incompatibles et sont à analyser en termes de stratégies d'identification.

Le sport, une quête d'assimilation

4 Dans une petite colonie sucrière, la vie économique et sociale est entièrement assujettie aux échanges imposés avec la Métropole. Les liaisons maritimes représentent un lien puissant, sorte de cordon ombilical qui relie et nourrit, apporte des modèles de pratiques inconnues, comme les pratiques sportives, et que leur origine valorise. Les relations avec la Métropole sont polarisées par l'idée d'un retard à combler. L'infériorité biologique affirmée au XIXème, y compris par des approches scientifiques s'est commuée en une échelle de développement. Dès lors, la colonisation n'est plus seulement l'exploitation d'un marché, mais se pare de prétendus devoirs. La France s'investit d'une mission de mise en valeur de ses colonies. Le développement du " capital humain » traduit bien la représentation de ces relations. Elle projette l'idée d'une hiérarchie dont l'éventuel comblement ne peut passer que par des voies "méritocratiques ». Cette vision, développée par l'école républicaine, magnifie tout ce qui vient de France, donnée comme symbole de Civilisation. Les pratiques physiques et sportives réservées au début du siècle à une caste privilégiée, sont rapidement investies d'un idéal qui les donne pour puissant moyen d'éducation, de promotion et donc d'intégration.

5 L'esprit sportif (Callède J-P., 1987) dans sa créolisation est avant tout un rejet. Le sport, résolument inscrit dans une recherche de modernité rompt systématiquement avec tout ce qui peut apparaitre comme stigmate d'une arriération. La culture physique ne s'épanouit que dans la distance avec les manifestations jugées grossières. Les statuts du club sportif moulien, par exemple, portent en 1932 l'exclusion de comportements indignes : " Sous peine d'être puni, il est totalement défendu d'injurier et expressément interdit de parler créole. » La langue marque une frontière. Pour accéder à la reconnaissance, il faut bannir tout ce qui est considéré comme marque d'archaïsme.

6 Le même règlement affiche un objectif clair "former et développer l'esprit sportif ", sans que celui-ci soit explicitement présenté ou détaillé. Il repose sur une connivence, une culture au sens où chaque membre de cette communauté en connaît implicitement les usages sans qu'ils aient fait l'objet d'un enseignement particulier. L'apprentissage se fait par imprégnation. L'analyse de la presse, recoupée de quelques enquêtes orales, permet de dresser une caractérisation de ces qualités partagées ou souhaitées. L'acclimatation des valeurs peut se lire à travers trois dimensions complémentaires qui caractérisent le sportif colonial : la mise en avant d'une culture physique, l'engagement public, la foi dans le progrès.

Une culture physique 
7 «Il est prouvé qu'on peut faire partie à la fois de l'élite physique et culturelle; qu'en somme l'élite tout court, la vraie élite ne peut se concevoir sans santé et force, complétant et rendant durable le plus bel épanouissement possible de l'esprit $»^{2}$. L'alliance dualiste classique se prolonge d'une sorte d'humanisme physique. Le " vrai » sportif est nécessairement un athlète complet qui rejette la spécialisation. Le but ultime n'est pas de gagner, mais de triompher des pesanteurs, de montrer une excellence, physique et morale. Ce qui est couronné, c'est une polyvalence au service de la santé, une force au service de l'utilité. "L'être fort, (...) est résistant, musclé, adroit, énergique, endurant et sobre. Il sait marcher, courir, sauter, grimper, lever des poids, en lancer, se défendre et nager. Un homme qui ne possède pas ces qualités n'est pas un citoyen complet $»^{3}$. Le sport est susceptible d'affermir, de discipliner, de cultiver; il apporte : « rectitude morale, instruction, robustesse du corps, vigueur du caractère $»^{4}$. L'image du gentleman, ou du " gentilhomme » est proposée en modèle, et peut s'y lire le processus de civilisation qu'ont caractérisé N. Élias et E. Dunning (1994). La « libération contrôlée des émotions » passe par l'intériorisation des mécanismes d'autocontrainte : «Etre fort c'est d'abord se vaincre soi-même »" Le self-control est signe de civilisation, et présenté comme condition de progrès social : « en toutes choses il doit $\mathrm{y}$ avoir une mesure. On ne saurait décemment sans risquer de porter atteinte aux droits du voisin franchir impunément les limites permises de la correction, de la courtoisie, de la modération de langage, de l'honnêteté de la discussion ${ }^{6}$. Cette incorporation de normes de bonne conduite valorise le spectacle sportif, leçon potentielle accessible à tous. Un ensemble de comportements est censé s'y donner en modèle. La correction vestimentaire s'accompagne d'un parfait contrôle de ses gestes, de ses attitudes, de ses émotions. Il est du rôle des "élus" de veiller à cette urbanité. Les spectateurs nécessitent d'être éduqués. On montre du doigt ceux qui envahissent le terrain, on expose les tables de la loi. «Dirigeants de clubs, n'oubliez pas que la bonne tenue du public fait partie de votre oeuvre éducative " 7 . Les joueurs doivent être guidés, afin "qu'ils se gardent de se laisser gagner par le chauvinisme de leurs supporters respectifs $»^{8}$. Fair-play, courtoisie et déférence envers les institutions policent les mœurs et doivent rendre compte d'un degré de civilisation.

L'esprit public

8 L'esprit sportif se constitue en Guadeloupe sur les valeurs de travail, d'effort gratuit, de dévouement pour le bien de la communauté. Le sportif ne peut se concevoir sans l'engagement au service de tous. Il y a une continuité de nature, en tout cas présentée comme telle entre sport et engagement social. "Notre devoir - le devoir de tous - est d'apporter, dans la mesure de nos facultés, quelque facteur de perfectionnement à l'existence collective $"$. La croyance sportive n'est pas à couper de l'engagement associatif et mutualiste ; l'influence socialiste et franc-maçonne est forte à cet égard. Le sport est un lieu d'investissement parmi d'autres. Les sportifs, engagés de fait dans la vie publique, sont appelés à prendre part aux responsabilités collectives. Au-delà des qualités énoncées, ce qui distingue le simple pratiquant du "parfait sportif ", c'est bien la mise au service d'un idéal. Les valeurs physiques ne se conçoivent que désintéressées, portées sur l'autel de la collectivité. L'exaltation de l'altruisme, du dévouement, rappellent que la naissance des pratiques sportives en Guadeloupe est étroitement associée au mouvement scout des Eclaireurs. Des objectifs sont solidement ancrés : " Sportifs guadeloupéens! A l'instar des Éclaireurs de France, que votre devise soit: servir! Toujours prêts! $»^{10}$. L'engagement se garde toutefois d'aller sur le terrain du politique. La neutralité est affirmée dans les règlements des clubs et associations, et maintes fois 
proclamée. Tout ce qui touche à la pratique politicienne est d'ailleurs entouré sinon de mépris, du moins de défiance. Mais si la coupure est annoncée avec tout engagement jugé partisan, le sportif est nécessairement un homme de la cité, et concerné par tout ce qui touche le citoyen. La position est clairement affirmée en Martinique. Pour Ferries Elizabeth, le rédacteur du journal Le Sportif, le sport ne se conçoit pas sans éducation intellectuelle, morale et citoyenne. "Nous ne nous occupons du corps que parce qu'il faut libérer l'esprit et nous nous occupons des questions sportives non comme un but mais comme un moyen. Mais rien de ce qui a rapport à la vie de la cité ne nous est indifférent. Rester en dehors des luttes politiques, d'accord, mais conserver intégralement nos droits de citoyens, dire notre mot chaque fois que besoin est, à propos de toute affaire concernant la cité $»^{11}$.

La foi dans le progrès

9 L'enjeu sportif est bien celui d'un avancement humain, de l'élévation dans une échelle fantasmée de valeurs, où l'Occident est donné et perçu comme référence. Le "parfait sportif» constitue ainsi le plus haut degré d'une hiérarchie, le citoyen abouti. Le classement ne passe pas par des qualités assignées ou héritées, mais par la preuve de capacités développées par le travail et l'effort, le renoncement, l'adhésion sans retenue à la modernité : il faut "avoir un esprit en éveil à l'affût de toutes les idées neuves et de tous

progrès ${ }^{12}$. Les formes en sont multiples et étroitement associées : perfectionnement corporel, par la culture physique et le sport, correction morale par la domination des instincts, progrès technique présenté comme la libération de l'homme, réforme sociale par la complète application des lois républicaines. "Nous Antillais, nous n'avons pas le droit de rester dans cette course rapide en retard sur l'horloge de l'histoire ${ }^{13}$. La thématique du retard est bien le lieu d'un enjeu fondamental: "Il semble que de mauvais génies s'emploient à travers les siècles, à entraver la marche de la Guadeloupe vers le progrès et la lumière ${ }^{14}$. Un des freins dans cette progression est la question de couleur qui heurte l'idéal sportif. "Il existe hélas entre nous de misérables mais tenaces préjugés de races, de couleurs et de classes " $^{15}$. La dénonciation de ce qui est désigné comme "la stagnation », est vigoureuse. Les références raciales sont soigneusement évitées. On insiste au contraire sur "l'entière et parfaite compréhension des hommes de toutes races " ${ }^{16}$. Le sport représente l'espoir de franchir les limites de l'assignation, les hommes devant être jugés sur leurs qualités et non sur leur pigmentation.

Un groupe engagé

10 "C'est aux plus instruits, aux plus éclairés qu'incombe le devoir de donner l'exemple $»^{17}$. Cette rhétorique copie à l'identique les rapports coloniaux. Le sportif fait partie d'une élite, et à ce titre, se doit de faire bénéficier le reste de la population de ses lumières : «Placé entre le standing d'une vie européenne qu'il comprend, qu'il a parfois assimilée, et une foule qui ne demande qu'à pouvoir, elle aussi réaliser les étapes nombreuses de cette ascension, l'intellectuel colonial n'est-il pas comme le guide? De lui, de ses directives, de ses suggestions doivent sortir la direction, la rapidité de cette marche vers la civilisation.

11 Il est le responsable sans s'être désigné. Toute la personnalité d'une contrée ou parfois d'un pays repose sur cette minorité. Elle sait la discrimination qui doit être faite entre les coutumes vieilles, usées, désuètes ne présentant aucune utilité, créant même parfois un dommage, et celles au contraire, qui par leur charme ou leur teneur, méritent d'être conservées, développées, vivifiées $»^{18}$. Qu'est ce donc qu'un «intellectuel colonial»? Issu des couches relativement aisées de la population, il a bénéficié d'une éducation longue, secondaire ou école normale, dispensée par la République, et par là même, accédé à des fonctions de service ou de responsabilité dans la colonie. Si le texte ne dit 
rien au sujet de la couleur, il laisse entendre d'autres filiations à cette «mission» : "Placé à mi-chemin des colonisateurs et des colonisés, venant des uns ayant rejoint les autres, ils doivent être l'interprète qui renseigne et explique ». Les mulâtres, surreprésentés dans l'enseignement secondaire, le sont aussi dans les fonctions publiques de la colonie que la République a multiplié, et auxquelles les études leur permettent d'accéder. Dès le début de la troisième République, les représentants de l'île sont, dans leur grande majorité des mulâtres. Ceux-ci se battent, s'appuyant sur une prétendue supériorité conférée par leur éducation, pour guider le peuple, lui tracer la voie. Le phénomène n'est pas propre à la Guadeloupe, et atteint de telles proportions dans les îles voisines que certains auteurs n'hésitent pas à parler de "mulâtrocratie ». Cette dominante mulâtre est réelle, mais les explications, par la copie d'un modèle blanc dont le métis chercherait toujours à se rapprocher, sont schématiques et insuffisantes. Elles restent en quelque sorte à l'épiderme des problèmes. Les limites et conditions de cette catégorisation raciale n'ont plus besoin d'être précisées (Bonniol J-L., 1990, 1992), mais la faiblesse de l'explication tient également à la réduction d'un phénomène complexe à une seule variable, la plus visible. La bourgeoisie citadine créole conjugue origines, itinéraires scolaires et professionnels, et foi dans un modèle de société qui a contribué à sa promotion. Au-delà de marqueurs phénotypiques, il s'agit bien d'une matrice commune de perceptions et d'actions. Que les mulâtres en soient les principaux représentants et bénéficiaires ne fait que renvoyer à l'intrication des déterminants dans la structure sociale de la colonie.

Identités culturelles

12 L'assimilation culturelle est flagrante. Le paysage ne s'imagine qu'en référence à des modèles métropolitains, les valses ouvrent les bals des sports, bref « l'élite » antillaise est nourrie d'une culture importée. Pourtant, la copie de modèles importés, le désir d'intégration ne signifie pas la disparition dans un ensemble mais bien au contraire, la participation, en pleine reconnaissance, à celui-ci. On ne compte plus les "contributions de la Guadeloupe à la pensée française ", les publications défendant l'idée d'une collaboration antillaise à l'œuvre de Culture. Les associations sportives participent de cette quête. Elles sont aussi culturelles et artistiques. Sur les 63 associations recensées en $1947^{19}$, il n'en est pas une qui ne soit multi-sports, et ne propose des activités littéraires ou esthétiques. Cette orientation est également sensible au niveau de la fédération de ces groupements, puisqu'ils se répartissent entre la tutelle des sports guadeloupéens (FSG) ou celle des associations culturelles de la Guadeloupe (FACG). La Solidarité Scolaire de Pointe à Pitre, fondée en 1917, agrège dès ses origines des activités de football, de gymnastiques et de théâtre. Elle est présentée comme «la doyenne des associations culturelles » lors du congrès de l'union sportive basse-terrienne (USBT) du 27 juillet 1947. De l'autre coté de l'île, Renaissance de StClaude, fondée en 1944, inscrit en championnat des équipes de football, basket et volley, où évoluent souvent les mêmes joueurs, que l'on retrouve au sein d'un petit orchestre, mais aussi dans l'animation d'évènements comme Les Floralies ou des projets d'aménagement des hauteurs de l'île. Plusieurs membres de cette association font partie du comité de rédaction du Dimanche sportif. Cette revue rend compte depuis sa création en 1945 d'activités extra-sportives. Dès le numéro 32 en novembre 1946 elle affirme: "en somme Le Dimanche sportif, qui demeure l'organe des sociétés sportives, devient également celui des groupements littéraires et artistiques. " Cette orientation est confirmée par le changement d'appellation en novembre 1947 où la revue devient Le Dimanche sportif et culturel, patronnée conjointement par la FSG et la FACG ${ }^{20}$. Ce journal, 
comme le clame son sous-titre, se veut "l'organe d'expression de la vie sportive et culturelle de la Guadeloupe $»^{21}$.

La Revue Guadeloupéenne est créée en janvier 1944. Publiée par le service d'information de la Guadeloupe et dépendances, elle comprend de nombreux sportifs dans son équipe, et sert d'ailleurs de caisse de résonance pour la promotion d'activités comme l'athlétisme, à l'initiative d'un jeune professeur d'éducation physique, Guy Azémar. Cette revue dès le numéro un de sa nouvelle série annonce « il s'agit moins d'informer les Guadeloupéens sur les événements de la France et du monde (...) que de faire connaître la Guadeloupe et de contribuer à son rayonnement $»^{22}$. L'attention s'est détournée, une existence est affirmée. "Il faut qu'à l'extérieur, on sache que la pénétration de la culture française n'a cependant pas détruit les coutumes, les traditions, les contes, les expressions, les chants, les danses, enfin tout ce qui forme le si pittoresque folklore créole ${ }^{23}$. Dans ce cadre, l'assimilation est clairement re-située : «Que m'importe le mot pourvu que nous n'en soyons pas dupes. Car l'assimilation c'est bien l'action d'assimiler et non d'être assimilé; c'est activité, c'est vie; non mort. M'assimiler des aliments c'est les transformer en mon sang (...) je me suis nourri de lait de chamelle dans mon enfance, et je ne sache pas que j'ai changé d'espèce, encore moins de sexe. Il n'en va pas autrement de l'esprit. Assimilons nous les cultures française, latine et grecque. Apprenons ainsi à nous découvrir et à nous exprimer. L'humanisme pour nous ne peut avoir d'autres sens. Se plonger jusqu'aux racines de notre race, écrit Mac Kay et bâtir sur notre propre fond, ce n'est pas retourner à l'état sauvage, c'est la culture même (...) et loin de tomber dans le racisme qui n'est que changer d'cillères en agissant ainsi, on ne peut que travailler à enrichir le patrimoine français, le patrimoine humain. C'est apporter notre part à l'œuvre de civilisation $»^{24}$. La copie d'un modèle ne s'envisage donc pas au détriment de la culture locale. L'après-guerre ancre la conviction qu'être égal ne veut pas dire être identique. Cette définition des frontières du même et du différent peut se lire à travers trois indices.

14 Le premier concerne la place des activités traditionnelles. La revue Paris-Match, dans son numéro 27 de décembre 1944 place les combats de coqs, si répandus aux Antilles parmi d'autres comptes-rendus, semblant les inclure de fait dans les activités sportives. Le Dimanche sportif voit dans cette pratique un pandémonium, emblème d'une perversion symbolisée par les paris et l'attitude qu'ils suscitent. Malgré tout, autour de la départementalisation, les activités traditionnelles semblent marquer un regain ou une émergence d'intérêt, comme si les tenants de la modernité redécouvraient les qualités de certaines pratiques familières. Les danses traditionnelles sont certes reléguées dans une approche "folklorique", mais les pratiques locales, danses, musiques, jeux traditionnels ne sont plus ignorées. Les luttes à l'aide de bâtons des mayolleurs permettent d'insister sur un "caractère sportif" et original: "Adresse, agilité, sûreté des mouvements, la souplesse des muscles, les parades, les attaques, les défenses? Tous éléments qui font honneur à la "race antillaise" "25. Ce qui change, c'est bien le regard porté sur soi-même. La culture ne se limite pas à un apport extérieur à imiter.

Le deuxième indice est la place du créole. Interdit par les clubs sportifs, inconnu dans Paris-Match, il est réservé dans Le Dimanche sportif à quelques rares plaisanteries ou blagues glanées sur le stade, c'est-à-dire maintenu à une place anecdotique. Il explose en décembre 1947 avec un " conte de chez nous ", tout en créole, et à suivre. Le journal attend plusieurs numéros avant de renouveler cette forme d'expression, et le sens s'en trouve peut-être affirmé. Elle marque l'inscription naturelle dans la langue maternelle. "Aujourd'hui la langue nationale entrée dans l'usage courant, n'a plus guère à redouter du 
patois et l'on peut sans crainte revenir à ce dernier pour le délassement, le divertissement, la détente $»^{26}$. Les publications utilisent de plus en plus ponctuellement le créole, ce qui suscite une ligne de démarcation. Ainsi, lors des jeux floraux des Antilles, les discours sont appréciés non plus seulement sur leur contenu, mais aussi sur leur support: On salue "l'auteur du premier discours en langue créole» tandis qu'il est rapporté que le promoteur de ces jeux lit son rapport en langue française ${ }^{27}$.

Le troisième indice émane des productions intellectuelles privilégiées. Encourageant le domaine littéraire, Le Dimanche sportif et culturel prévoit des prix pour récompenser :

- « $1^{\circ}$ les élèves des écoles

- $2^{\circ}$ les étudiants guadeloupéens

- $3^{\circ}$ des Antillais »

17 À travers trois strates d'âge, le but est bien d'encourager une production locale. Celle-ci peut exprimer une solide acculturation, peu importe le thème ou l'approche, il est trop tôt pour cette complète bascule. Mais le centre de gravité s'est déplacé, du modèle extérieur, vers celui qui localement produit. Dans le même esprit, des concours de monographies antillaises sont organisés : «La première qualité du sujet sera son originalité, sa couleur locale. Les concurrents devront se limiter dans le choix de leur sujet au plan guadeloupéen. Ils devront essayer soit de mettre en lumière autant que possible un des usages folkloriques guadeloupéens, soit de relater un conte, une légende, une nouvelle guadeloupéenne, avec des personnages guadeloupéens, dans un cadre guadeloupéen ${ }^{28}$.

Une existence et une différence osent s'affirmer. L'idée d'une culture antillaise jaillit, éclaboussant la problématique de l'égalité. Dès lors, la référence exclusive à un modèle importé va progressivement se doter d'un statut négatif. En se spécifiant, la notion d'assimilation culturelle change de polarité. L'idée sportive, avec ses ramifications, oscille entre désir d'intégration et affirmation d'une particularité. Mais le ressort principal en demeure la quête de reconnaissance : «On n'a répandu le préjugé d'infériorité dans notre propre crâne que pour mieux nous exploiter. Les faits démentent en tous points le préjugé exécrable. Notre bravoure à la guerre est légendaire. Nous avons montré dans les sports que nous sommes aussi forts et aussi sains que les autres races de la terre $»^{29}$.

19 Le succès même du terme métissage invite à la prudence. Aujourd'hui métaphore de la rencontre culturelle, il est paré d'une valence positive au point de désigner un idéal de relation, qui déborde très largement les cadres biologiques d'origine. Pourtant le maniement sans précautions de cette notion présuppose celle d'entités voire d'essences, et néglige les processus d'identification, les constructions alternatives, cumulatives, fantasmatiques (Fuma S., Poirier J., 1992). Au niveau culturel, mixophilie ou mixophobie (Amselle J-L., 1996) sont bien les deux faces d'une même médaille. Elles continuent de penser le métissage comme perte ou gain, autrement dit de le placer d'office dans une hiérarchie latente de valeurs. 


\section{BIBLIOGRAPHIE}

Amselle J-L. (1996) Vers un multiculturalisme français, Paris, Aubier.

Bonniol J-L. (1990) La couleur des hommes comme principe d'organisation sociale, Ethnologie

française, 4, 410-418.

Bonniol J-L. (1992) La Couleur comme maléfice, Paris, Albin Michel

Callède J-P. (1987) L'Esprit sportif, Essai sur le développement associatif de la culture sportive, Presses Universitaires de Bordeaux.

Elias N., Dunning E. (1994) Sport et civilisation. La violence maîtrisée, Paris, Fayard (1986).

Fuma S, Poirier J. (1992) Métissages, hétéroculture et identité culturelle, in Métissages tome 2, linguistique et anthropologie s/d URA 1041 CNRS, Paris, L'Harmattan.

Turgeon L., Delage D., Ouellet R. (1996) Transferts culturels et métissages, Amérique/Europe, Paris, L'Harmattan.

\section{NOTES}

1. Voir à ce sujet les comptes rendus des séances du conseil général du début du XXe.

2. Le Dimanche sportif et culturel, 99, 16 mai 1948.

3. Le Dimanche sportif et culturel, 77, 14 décembre 1947, éditorial.

4. Le Dimanche sportif et culturel 130, 30 mars 1952.

5. Le Dimanche sportif et culturel, 112, 28 novembre 1951.

6. Le Dimanche sportif, 17, 7 avril 1946.

7. Le Dimanche sportif et culturel, 122, 3 février 1952, encadré p. 98.

8. Clartés, 4, 6 octobre 1945 (journal catholique).

9. Le Dimanche sportif et culturel, 128, 16 mars 1952, Propos de la semaine, P. Baptistide.

10. Le Dimanche sportif et culturel, 75, 30 novembre 1947, p. 340.

11. Le Sportif, 357, 11 mai 1946.

12. Le Dimanche Sportif, 70-71, 3-10 août 1947.

13. Revue Guadeloupéenne, 40, avril mai juin 1960, «Etre réaliste (en guise d'éditorial) » de B. Lara.

14. Le Dimanche sportif et culturel, 75, 30 novembre 1947, éditorial

15. Paris Match 109, 1er avril 1949.

16. Le Dimanche sportif, 47, 23 février 1947.

17. Le Dimanche sportif, 19, 20 avril 1946, Propos du sportif.

18. Le Nouvelliste de la Guadeloupe, samedi 19 février 1938.

19. CARAN(Paris), F17/14987, CAOM (Aix en Provence), FOM 112, dossier 46.

20. Ce qui permet à la FACG, dans sa demande de subvention au ministère d'inclure dans ses activités la réalisation d'un bulletin hebdomadaire et d'un supplément mensuel, Le Dimanche culturel. CARAN, F44/75.

21. Les changements de tutelle et d'orientation rappellent toutefoids les crises au sein du mouvement sportif. Ainsi la FSG retire en 1948 son patronage au Dimanche sportif et culturel. A partir de mars 1952, le journal devient : Bulletin de propagande en faveur du sport et de la culture populaire en Guadeloupe. 
22. Revue guadeloupéenne, $\mathrm{n}^{\circ} 1$ (nouvelle série), septembre 1945.

23. Ibid.

24. Revue guadeloupéenne « Léopold Senghor et l'humanisme noir », 5, mars - avril 1946, p. 24-25.

25. Le Dimanche sportif, 7, 27 janvier 1946, p.52 article signé Solange. Seule occurrence rencontrée de cette « race antillaise » qui dit en tout cas la fierté et la singularité de l'appartenance.

26. R. Nainsouta, Revue guadeloupéenne, 10, janvier - février 1947.

27. Revue Guadeloupéenne, 23-24, septembre - octobre 1949.

28. Revue guadeloupéenne, 10, janvier - février 1947.

29. Europe Colonies, 21 juillet 1945, p.13. Jules Alcande, responsable de cette publication et signataire de cet article est Guadeloupéen, par ailleurs surveillé par les renseignements généraux pour ses propos jugés séditieux.

\section{RÉSUMÉS}

Cet article, basé sur l'analyse de la presse, examine la croyance dans les vertus du sport dans une petite colonie devenant département français. La soif d'être assimilés, c'est-à-dire reconnus comme des citoyens français à part entière, fait de l'esprit sportif un fort vecteur d'acculturation. Pourtant, support d'une intégration, le sport est aussi le lieu d'affirmation d'une différence.

This paper, based on press analysis, examines the belief in virtues of sport in a small colony becoming a french departement. The yearning to be assimilated, to become « fully fledged french citizens » brings. the sporting spirit as a strong medium toward acculturation. But, support for integration, sport is also a place to show the right to be different.

\section{INDEX}

Keywords : assimilation, colonies, cultural identity, equality, sporting spirit

Mots-clés : esprit sportif, égalité, identité culturelle

\section{AUTEUR}

\section{JACQUES DUMONT}

Laboratoire Archéologie industrielle, histoire et patrimoine. Université des Antilles et de la Guyane 